\title{
Determination of non-uniform settlements caused by decompression of soil in the excavation
}

\author{
Viktor Yarkin $^{1 *}$, Anna Kukhar ${ }^{1}$ \\ ${ }^{1}$ Donbas National Academy of Civil Engineering and Architecture, 2, Derzhavina Str., Makeyevka, \\ DPR, 286123
}

\begin{abstract}
The article is offered refined and simplified methods for determination of non-uniform settlements caused by decompression of soil in the excavation. The results of the calculations executed taking into account the done suggestions and in the program Plaxis are presented. The results of the calculations are compared with the results of experimental observations given in the literature. The influence of the excavation parameters on the non-uniform settlements is investigated. Present a proposal for adjustment of the conditions, which require consideration of soil decompression in the excavation.
\end{abstract}

\section{Introduction}

At excavations as a result of removing the load from the weight of the extracted soil, the lower layers of soil undergo decompression and, as a consequence, the bottom of the excavation is lifted. The lifting of bottom of the excavation depends on its depth and sizes in plan, texture and mechanical characteristics of soils, the presence of enclosing structures of the excavation, the duration of work on the excavation and the construction of the building, etc. [1, 2].

Since the decompression of the soil massif along the depth in the central part of the excavation will be larger than at the edges (Fig.1b), then under subsequent loading it will give a large settlement, which in turn can lead to additional small but non-uniform settlements with a tendency to the deflection of the building [3, 4].

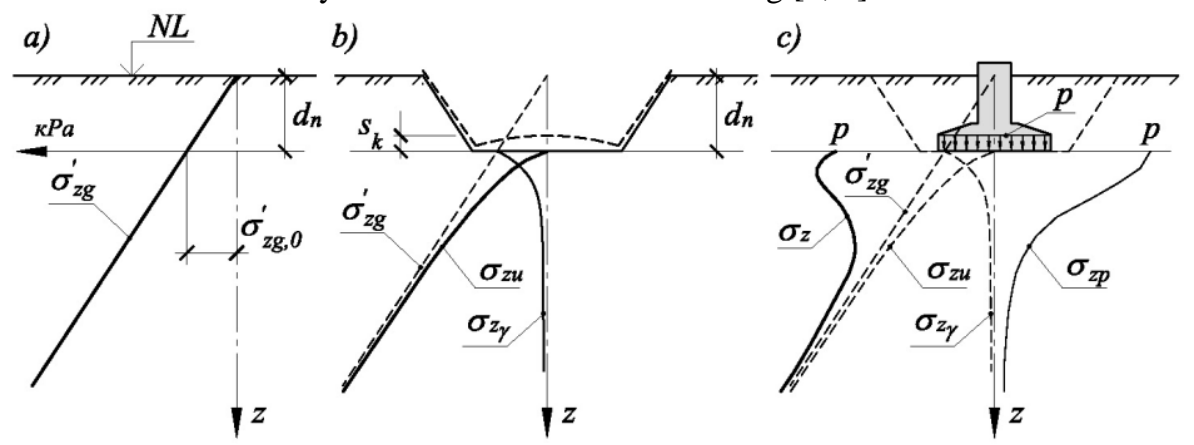

Fig. 1. Stress diagrams in a soil massif: $a$ - in a natural massif; $b$ - after excavation; $c$ - after loading pressure base foundation

When constructing foundations in the excavation differentiate the following values of vertical stresses:

- from dead weight pressure of soil before construction begins $\sigma_{z g}^{\prime}$ (Fig. 1a);

- from dead weight pressure of soil extracted from the excavation $\sigma_{z \gamma}($ Fig. 1b);

- from dead weight pressure of soil after excavation $\sigma_{z u}$ (Fig. 1b);

*Corresponding author: YarkinVV@mail.ru 
- from dead weight pressure of soil after the construction of the building $\sigma_{z g}$;

- from external load after the construction of the building $\sigma_{z p}$ (Fig. 1c);

- total after the construction of the building $\sigma_{z}$ (Fig. 1c).

In ordinary engineering-geological conditions, non-uniform settlements caused by decompression of soil in the excavation usually does not have a significant effect on the stress-strain state (SSS) of building constructions. In this regard, the current regulatory documents for design of soil bases and foundations $[5,6]$ allow to ignore the settlements from decompression at a depth of excavation of less than $5 \mathrm{~m}$. However, in difficult engineering-geological conditions, when under the influence of various factors, the occurrence of significant uneven deformations of the base may occur, consideration of the settlements caused by decompression of soil can both reduce the overall unevenness and aggravate it $[7,8]$.

Recently, numerical methods for determining the SSS of a soil massif with the help of specialized geotechnical software using the finite element method (FEM) [2, 3, 9] and various elastic and elastic-plastic models of the soil base have become increasingly popular $[10,11,12,13,14,15]$. Nevertheless, analytical methods of calculation and at the moment have not lost relevance, since they are most fully developed in regulatory documents $[5,6]$ for almost all types of difficult engineering-geological conditions, are well confirmed by experimental data $[16,17]$ and are one of the ways verification of the results of numerical calculation methods. A widely used method of layer-by-layer summation to determine the settlement of the bases continues to be improved [18] and by applying the deformation characteristics of the soil, which are determined taking into account its stress state $[19,20]$, it is possible to significantly improve the accuracy of the determination of the settlement.

It should be noted that the formula (1) used in the current regulatory documents $[5,6]$ at the size of the excavation significantly exceeding the size of the foundation has a certain incorrectness caused by taking into account the unloading of the bases during excavation.

$$
s=\beta \cdot \sum_{i=1}^{n} \frac{\left(\sigma_{z p, i}-\sigma_{z \gamma, i}\right) \cdot h_{i}}{E_{i}}+\beta \cdot \sum_{i=1}^{n} \frac{\sigma_{z \gamma, i} \cdot h_{i}}{E_{e l, i}}
$$

where $\beta$-non-dimensional coefficient, equal 0.8 ;

$E_{i}$ - deformation modulus of the $i$-th elementary layer of soil across the branch of primary loading;

$E_{e l, i}$ - deformation modulus of the $i$-th elementary layer of soil across the branch of secondary loading (modulus of elasticity);

$\sigma_{z p, i}-$ average value vertical stress from external load in the $i$-th elementary layer of soil;

$\sigma_{z \gamma, i}-$ average value vertical stress from dead weight pressure of soil extracted from the excavation in the i-th elementary layer of soil on the vertical, passing through the center of the basement;

$h_{i}$ - height of the $i$-th elementary layer;

$n$ - number of elementary layers located within the compressible stratum.

When using this formula under certain conditions, for example, with the surface of the planning practically coinciding with the surface of the natural relief, the great depth of excavation and the size of the excavation, which substantially exceed size of the foundation, in the layers of the lower zone of the compressible stratum may arise negative vertical stresses from the external load.

In this case, the negative settlements of these elementary layers are not fully compensated by a component that takes into account the decompression of the soil during the excavation. An increase in the compressible stratum, caused, for example, by the influence of neighboring foundations, in this situation leads to a paradoxical decrease of the settlements. As a result, the settlement, determined in accordance with $[5,6]$, is significantly less than in accordance with [21].

Previously, this incorrectness was eliminated by zeroing the stresses $\sigma_{z \gamma, i}$, if they exceeded the stresses $\sigma_{z p, i}(\S 2.231$ [22]), however, in the current regulatory documents this indication is absent. 
Consider the formula (1) in the following form

$$
s=\beta \cdot \sum_{i=1}^{n} \frac{\left(\left(p_{0}+\sigma_{z g, 0}^{\prime}\right) \cdot \alpha_{i}-\sigma_{z g, 0}^{\prime} \cdot \alpha_{i}^{\prime}\right) \cdot h_{i}}{E_{i}}+\beta \cdot \sum_{i=1}^{n} \frac{\sigma_{z g, 0}^{\prime} \cdot \alpha_{i}^{\prime} \cdot h_{i}}{E_{e l, i}}
$$

where $p_{0}=p-\sigma_{z g, 0}^{\prime}-$ additional pressure in the level of the foundation base;

$p$ - average pressure on the foundation base;

$\sigma_{z g, 0}^{\prime}$ - vertical stress from dead weight pressure of soil extracted from the excavation in the level of the foundation base;

$\alpha_{i}$ - coefficient that takes into account the distribution of stresses from external load in depth, depending on the size of the foundation;

$\alpha_{i}^{\prime}$ - coefficient that takes into account the distribution of stresses from the dead weight pressure of soil extracted from the excavation, in depth, depending on the size of the excavation.

Under the conditions described above, with increasing depth, the damping of the stresses from the external load $\left(p_{0}+\sigma_{z g, 0}^{\prime}\right) \cdot \alpha_{i}$ will be more intense than that stresses from the dead weight pressure of soil extracted from the excavation $\sigma_{z g, 0}^{\prime} \cdot \alpha_{i}^{\prime}$ (Fig. 2).

This situation is possible in the absence of backfilling of the excavation, but then the component taking into account the decompression of the soil overstates the settlement, since in it the load from the dead weight of the soil above the foundation base is accepted acting throughout the area of the excavation.

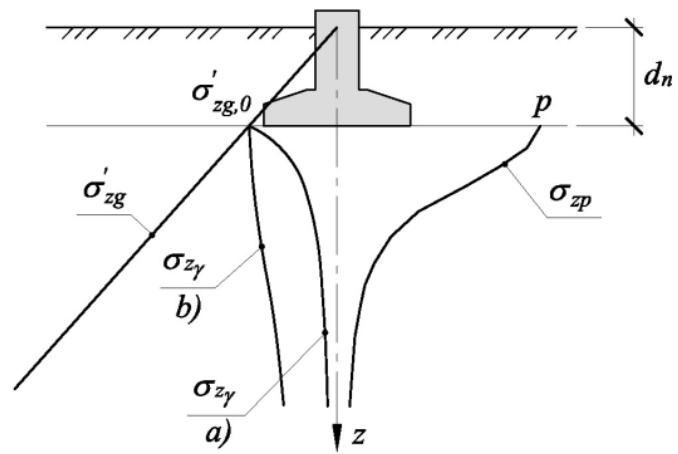

Fig. 2. The scheme of distribution of stresses in the base of the foundation: a - the sizes of the foundation in the plan are comparable with the sizes of the excavation $b$ - the size of foundation in the plan are an order of magnitude smaller than the size of the excavation

Another disadvantage of formula (1) is the dependence of the component, taking into account the decompression of the soil, on the compressible stratum determined for a particular foundation.

Depending on the ratio of mean pressure and vertical stress from the dead weight pressure of soil extracted from the excavation $p / \sigma_{z g, 0}^{\prime}$, according to the norms $[5,6]$, the calculated compressible stratum can be either larger or smaller than the actual zone of soil decompression.

Analysis of experimental observations of stratified decompression deformations shows that the bulk of the deformations are realized in the upper zone of the unloaded base. About $90 \%$ of the elastic deformation occurs within a depth slightly exceeding the size of the unloaded area [23].

\section{Methods}

To eliminate these incorrectness, it is proposed to present the total settlement in the form of separately determined components from additional vertical stresses and elastic deformations caused by unloading the foundation during the excavation, with an appropriate division into elementary layers.

$$
s=s_{p}+s_{k}
$$




\section{EECE-2018}

where $s_{k}$ - settlement at a pressure equal to natural pressure, determined using deformation modulus of soil across the branch of secondary loading;

$s_{p}$ - settlement with a further increase in pressure to its full value, determined using deformation modulus of soil across the branch of primary loading.

$$
s_{p}=\beta \cdot \sum_{i=1}^{n} \frac{\left(\sigma_{z, i}-\sigma_{z g, i}^{\prime}\right) \cdot h_{i}}{E_{i}}=\beta \cdot \sum_{i=1}^{n} \frac{\sigma_{z p, i} \cdot h_{i}}{E_{i}}
$$

where $\sigma_{z p, i}=\alpha_{i} \cdot p_{0}$-additional vertical stresses at a depth $z_{i}$ from the foundation base;

the remaining notations are the same as in formulas $(1-2)$.

Settlement, caused by the unloading of the foundation during excavation, during subsequent loading:

- of the whole area of the excavation by pressure $p \geq \sigma_{z g, 0}^{\prime}$

$$
s_{k}=\beta \cdot \sum_{i=1}^{n} \frac{\sigma_{z \gamma, i} \cdot h_{i}}{E_{e l, i}}
$$

where $n$ - number of elementary layers located within the depth of the unconsolidated zone of the soil;

- of the whole area of the excavation with uniform pressure $p<\sigma_{z g, 0}^{\prime}[24]$

$$
s_{k}=\beta \cdot \sum_{i=1}^{n} \frac{\sigma_{z \gamma, i} \cdot h_{i}}{E_{e l, i}} \cdot \frac{p}{\sigma_{z g, 0}^{\prime}}
$$

- non-uniform pressure $p<\sigma_{z g, 0}^{\prime}$

$$
s_{k}=\beta \cdot \sum_{i=1}^{n} \frac{\sigma_{z a, i} \cdot h_{i}}{E_{e l, i}}
$$

where $\sigma_{z a, i}$ - vertical stress along the vertical axis considered, taking into account the influence of all differently loaded areas within the bottom of the excavation.

As a limitation of the depth of the unconsolidated zone of the soil take condition $\sigma_{z, i} \leq k \cdot \sigma_{z g, i}$, where $k$ accepted 0.2-0.5 depending on the width of the excavation.

Graphically, the dependence of the settlement of the $i$-th elementary layer of soil on the stresses acting in it is shown in Fig. 3.

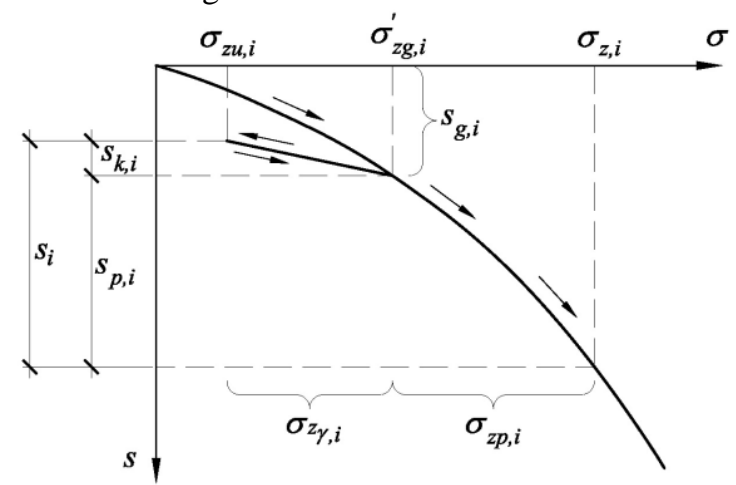

Fig. 3. Dependence of the precipitation of the $i$-th elementary layer on the stresses acting in it: $s_{g}-$ settlement from the action of its own weight of the soil, which occurred during the formation of the soil massif

Non-uniform settlements caused by decompression of soil during the development of the excavation is determined taking into account the location of foundations in the excavation.

Vertical stress from dead weight pressure of soil extracted from the excavation taking into account the location of the calculated vertical in the excavation plan is determined by the formula 


$$
\sigma_{z \gamma, i}=\alpha_{i}^{\prime} \cdot \sigma_{z g, 0}^{\prime}
$$

where $\alpha^{\prime}$ - coefficient taking into account the distribution of stresses over the depth, determined by the method of corner points along the calculated vertical line passing through the center of the foundation base, depending on the size of the excavation and the location of the calculated vertical (Fig. 4);

$$
\begin{gathered}
\alpha_{i}^{\prime}=0.25 \cdot\left(\alpha_{1, i}+\alpha_{2, i}+\alpha_{3, i}+\alpha_{4, i}\right)=0.25 \cdot \sum_{j=1}^{4} \alpha_{j, i} \\
\alpha_{j, i}=\frac{2}{\pi} \cdot\left(\operatorname{arctg} \frac{\eta_{j}}{\xi_{j, i} \cdot \sqrt{\xi_{j, i}^{2}+\eta_{j}^{2}+1}}+\frac{\eta_{j} \cdot \xi_{j, i} \cdot\left(2 \cdot \xi_{j, i}^{2}+\eta_{j}^{2}+1\right)}{\left(1+\xi_{j, i}^{2}\right) \cdot\left(\eta_{j}^{2}+\xi_{j, i}^{2}\right) \cdot \sqrt{\xi_{j, i}^{2}+\eta_{j}^{2}+1}}\right)
\end{gathered}
$$

$\eta_{j}=l_{j} / b_{j}$ - aspect ratio of the $j$-th area of the excavation;

$\xi_{j, i}=z_{i} / b_{j}$ - relative depth for the $j$-th area of the excavation.
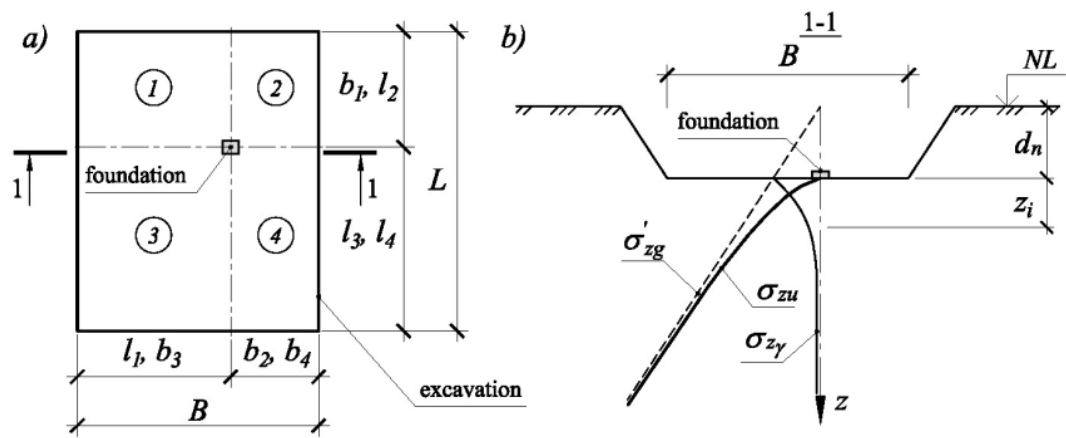

Fig. 4. Schema for determining $\sigma_{z \gamma}$ : a - plan, b-cross section

\section{Results and Discussion}

The results of measuring lifting of the excavation bottom with natural slopes of $10 \mathrm{~m}$ depth with dimensions in the plan of $47.7 \times 86 \mathrm{~m}$ at three points are given in [25]: at the center of the excavation and at distances from the center $\approx 9 \mathrm{~m}$ and $\approx 20 \mathrm{~m}$. In this work, for the indicated parameters of the excavation, lifting of the excavation bottom was determined at these points according to the proposed method and in the Plaxis software (Fig. 5). Numerical modeling in Plaxis is performed in a flat formulation of the problem using the model Hardening Soil (HS) as the most suitable for performing such calculations [11, 13, 26, 27].

Modules of elastic deformation of soil layers were adopted from the condition of ensuring the coincidence of the lifting in the center of the excavation.

Design parameters of soil:

- for the model elastic half-space: $\gamma=19 \mathrm{\kappa N} / \mathrm{m}^{2} ; E=25.5 \mathrm{MPa} ; E_{e l}=76.5 \mathrm{MPa}$;

- for the model HS: $\gamma_{\text {sat }}=20 \mathrm{KN} / \mathrm{m}^{2} ; \gamma_{\text {unsat }}=19 \mathrm{\kappa N} / \mathrm{m}^{2} ; v=0.3 ; C=50 \mathrm{kPa} ; \varphi=20^{\circ} ; E_{\text {oed }}=25.5$ $\mathrm{MPa} ; E_{50}=25.5 \mathrm{MPa} ; E_{u r}=76.5 \mathrm{MPa}$.

\begin{tabular}{|c|c|c|c|c|c|c|}
\hline \multirow{2}{*}{$\begin{array}{l}\text { Distance from the } \\
\text { center to the point, } \mathrm{m}\end{array}$} & \multicolumn{2}{|c|}{ Experimental } & \multicolumn{2}{|c|}{ The proposed method } & \multicolumn{2}{|c|}{ Plaxis (HS model) } \\
\hline & $s_{k}, \mathrm{~mm}$ & $\Delta s_{k} / L$ & $s_{k}, \mathrm{~mm}$ & $\Delta s_{k} / L$ & $s_{k}, \mathrm{~mm}$ & $\Delta s_{k} / L$ \\
\hline 0 & 36.2 & \multirow{2}{*}{0.00022} & 36.2 & \multirow{2}{*}{0.00029} & 36.02 & \multirow{2}{*}{0.00017} \\
\hline 9 & 34.2 & & 33.6 & & 34.5 & \\
\hline 20 & 21.8 & 0.00113 & 20.7 & 0.00117 & 24.6 & 0.0009 \\
\hline
\end{tabular}

The results are summarized in the table 1 .

Table 1. Deformations of the bottom of the excavation 


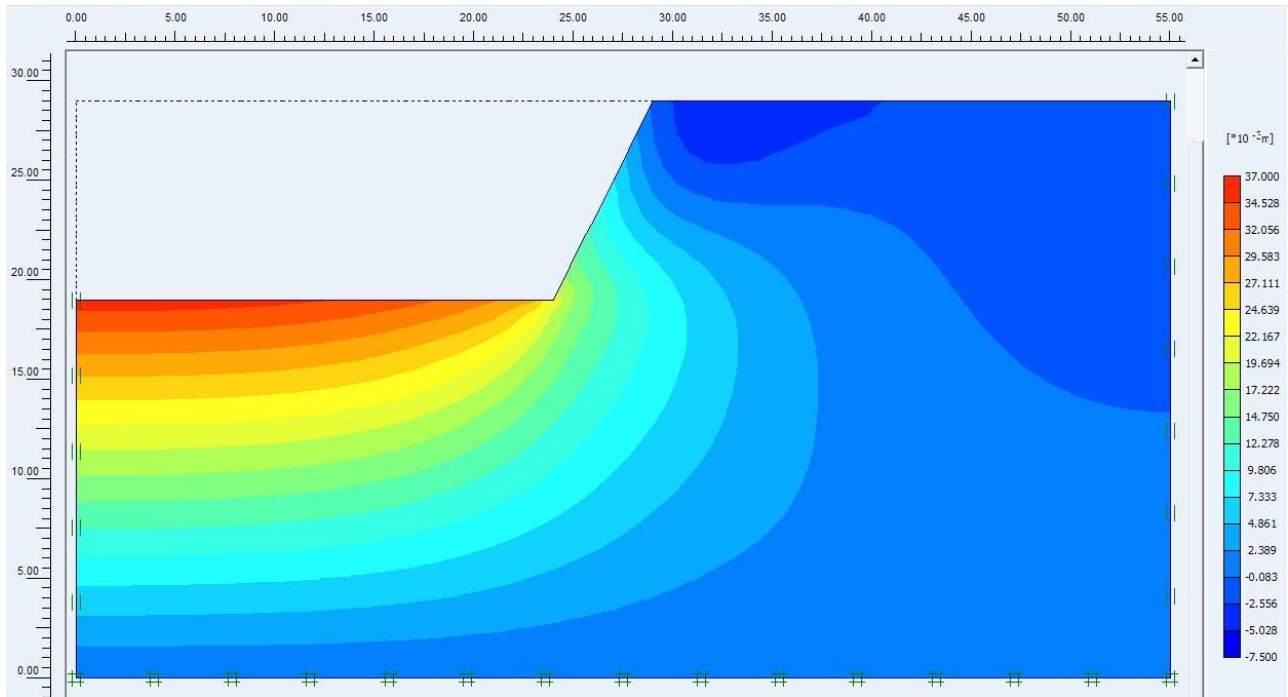

Fig. 5. Vertical displacements soil massif

The nonuniformities of the settlements obtained by proposed method and in Plaxis showed good convergence with the experimental values. The proposed method using the elastic half-space model overestimated the nonuniformities, and Plaxis with the HS model understated it. The value of the unconsolidated zone was also smaller in the Plaxis with the HS model

Also, in accordance with the proposed method, was investigated the influence of such factors as the parameters of the excavation (sizes in plan and depth) (Fig. 6) and the mechanical properties of the soil (density and modulus of elastic deformations) on the scheme of non-uniform settlements from soil decompression.

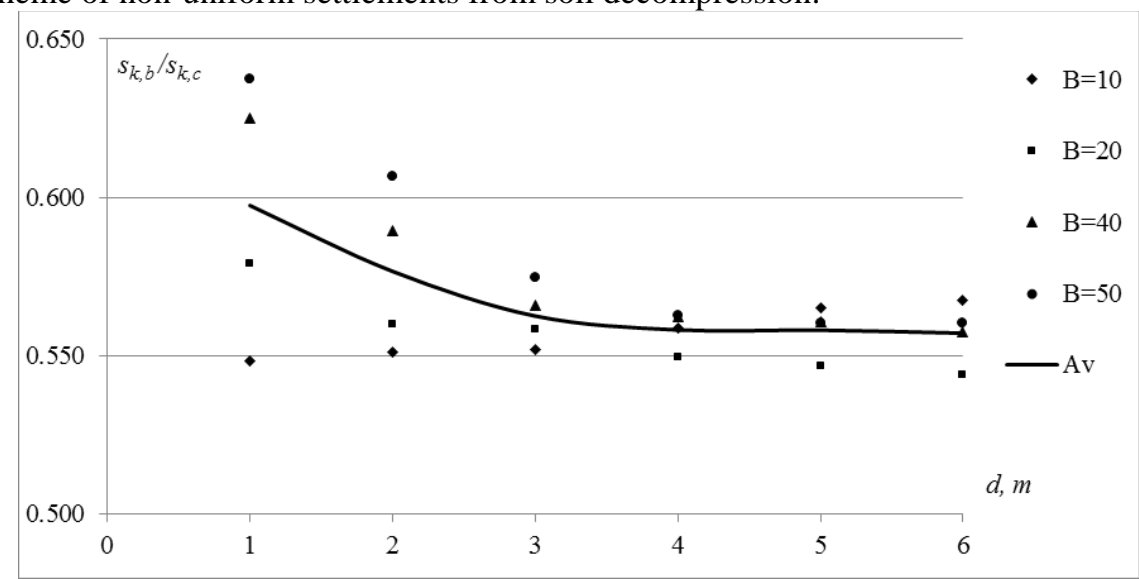

Fig. 6. Ratio of the settlements of points on the edge and in the center of the excavation bottom at various parameters of the excavation

The results obtained showed that for the excavations with depth of $\mathrm{d}>3 \mathrm{~m}$, with a uniform bedding in the base and natural slopes, the ratio of the settlements of points on the edge $s_{k, b}$ and in the center $s_{k, c}$ of the excavation bottom, varies in the range $0.545-0.57$ (mean the value is $\approx 0.56$ ). With an almost constant ratio of settlements of points on the edge and in the center of the excavation bottom, increasing the size of the excavation leads to a reduction in the non-uniform settlements. 
Non-uniform settlements from decompression of soil in the outer zones of the excavation is much higher than in the central part. To describe the scheme of non-uniform settlements from soil decompression with relatively uniform soil bedding, can take the conditional radius of curvature, defined by formula

$$
R=\frac{B^{2}}{8 \cdot\left(s_{k, c}-s_{k, b}\right)} \approx \frac{B^{2}}{8 \cdot(1-0.56) \cdot s_{k, c}}=\frac{B^{2}}{3.52 \cdot s_{k, c}}
$$

where $s_{k, c}$ and $s_{k, b}$ - settlement from decompression of soil, respectively, of points in the center and on the edge of the excavation bottom; $B$ - width of excavation.

In this case, the settlement from the decompression of the soil at the point of the crosssection of the excavation located at a distance $x$ from the center can be found from

$$
s_{k, x}=s_{k, c}-\frac{x^{2}}{2 \cdot R}
$$

The error of the non-uniform settlements, determined from formulas $(10,11)$ with respect to the experimental data given in Table 1, does not exceed 5\%.

\section{Conclusions}

The proposed method for determining the unevenness of settlements caused by soil decompression allows:

- regardless of the size of the foundation for the given parameters of the excavation, determine the maximum settlement from the decompression of the soil in the center of the excavation;

- determine by the simplified formulas $(10,11)$ the non-uniform settlements caused by the decompression of the soil with sufficient accuracy to make a decision on the need to take it into account;

- if necessary, refine the non-uniform settlements by using formulas (5-9).

In current regulatory documents for the design of base of foundations, the decompression of the soil in the excavation is allowed to be ignored at a depth of excavation less than $5 \mathrm{~m}[6,5]$. However, according to the authors, under certain conditions other criteria should be used:

- for buildings that are particularly sensitive to non-uniform settlements in difficult geotechnical conditions can be ignored if the conditional radius of curvature, defined by formula (10), $R \geq 20 \mathrm{~km}$;

- for dense clay soils with explicit elastic properties at the base it is proposed to use the condition

$$
\frac{\sigma_{z g, 0}^{\prime} \cdot \bar{E}}{\sigma_{z, 0} \cdot \bar{E}_{e l}} \leq 0.1
$$

where $\sigma_{z, 0}$ - total vertical stress in the level of the foundation base after the construction of the building; $\bar{E}, \bar{E}_{e l}$ - respectively, the averaged values of the modulus of the total and elastic deformation within the zone of soil decompression, which in the first approximation can be taken equal to the width of the excavation.

\section{References}

1. O. Shulyatyev, Bases and foundations of high-rise buildings (2016)

2. H. Faheem, F. Cai and K. Ugai, Computers and Geotechnics, 31(2) Pp. 67-74 (2004)

3. R. Finno, J. Blackburn, and J. Roboski, Journal of Geotechnical and Geoenvironmental Engineering, 133 (1) (2007)

4. R. Fuentes, Geotechnical Research, Pp. 1-12 (2018)

5. SP 22.13330.2016. The foundations of buildings and structures (2016) 
6. DBN B.2.1-10-2009. Bases and foundations of buildings and structures (2009)

7. B. Garagash, Reliability of spatial regulated systems "soil base - structure" with uneven deformations of the base, 1 (2012)

8. M. Schuster, C. Juang, M. Roth, and D. Rosowsky, Géotechnique, 58 (9) Pp. 743749 (2008)

9. L. Zradkovic, D. Potts and St. John HD, Géotechnique, 55(7) Pp. 497-513 (2005)

10. A. Bespalova, A. Bespalov, A. Karabanov, A. Ter-Martirosyan, Vestnik MGSU, 2 Pp. 119-128 (2008)

11. I. Yudina, A. Klimov, Vestnik MGSU, 2 Pp. 145-149 (2008)

12. M. Bezgodov, S. Kaloshina, Bulletin PNRPU. Construction and architecture, 2 (6) Pp. 17-27 (2012)

13. A. Ponomarev, S. Kaloshina, A. Zakharov, M. Bezgodov, R. Shankman, D. Zolotozubov, Bulletin PNRPU. Construction and architecture, 4 Pp. 189-202 (2014)

14. R. Mangushev, N. Nikiforova, Technological settlements of buildings and structures in the zone of influence of underground construction (2017)

15. A. Ponomarev, A. Zakharov, D. Zolotozubov, S. Kaloshina, Bases and foundations (2015)

16. V. Ledenev, A. Khudyakov, Mechanical and rheological models of bases and foundations (2012)

17. V. Ledenev, Bases and foundations at complex force influences (experiments), $\mathbf{3}$ (2017)

18. V. Utkin, Mag. Civ. Eng., 1 (61) Pp. 4-13 (2016)

19. D. Rachkov, Ya. Pronozin, V. Chikishev, Mag. Civ. Eng., 4 (72) Pp. $36-45$ (2017)

20. Yu. Kalugina, D. Keck, Ya. Pronozin, Mag. Civ. Eng., 7 (75) Pp. 139-149 (2017)

21. SNiP 2.02.01-83. Foundations of buildings and structures (1985)

22. Manual on the design of the bases of buildings and structures (to SNIP 2.02.0183) (1986)

23. V. Ulitsky, A. Shashkin, K. Shashkin, Geotechnical support of urban development (2010)

24. S. Klepikov, Calculation of structures on a deformable base (1996)

25. O. Shulyatyev, Bulletin PNRPU. Construction and architecture, 4 Pp. 203-245 (2014)

26. L. Strokova, Bulletin of Tomsk Polytechnic University, 313(1) Pp. 69-74 (2008)

27. O. Sokolova, Mag. Civ. Eng., 4 Pp. 10-16 (2014) 